\title{
Interrogating Systems That Cause Disparities: Testing the Social-Ecological Model in Low- Versus High- Density African American Communities
}

\author{
Kaprea F. Johnson, $\mathrm{PhD}$
}

The Ohio State University, Columbus, Ohio, United States

(iD) https://orcid.org/0000-0001-7127-3370

Lauren B. Robins, $\mathrm{PhD}$

Old Dominion University, Norfolk, Virginia, United States

(iD) https://orcid.org/0000-0002-8898-3499

Chelsea L. Williams, $\mathrm{PhD}$

Virginia Commonwealth University, Richmond, Virginia, United States

(iD https://orcid.org/0000-0002-5244-8822

Tiffany G. Townsend, PhD

Augusta University, Augusta, Georgia, United States

Contact:johnson.9545@osu.edu

\section{Abstract}

This study utilized the four-level social-ecological model to provide a better understanding of the disparities in health-related outcomes in high- and low-density African American (AA) communities. The current research sought to understand the relationships between mental and physical health, social-economic factors, and physical environment within this community. The goal of this study was to understand the relationship between these indicators of health, to better inform health-care strategies. The results highlight the significant difference between high- and low-density AA communities and socio-economic factors, physical environment, poor physical days, and poor mental health days. Implications for behavioral health providers are explored.

Keywords: social ecological model (SEM), African American (AA), African American community population density (AACPD), high-density, behavioral health providers

Date Submitted: April 1, 2020 | Date Published: December 2, 2021

\section{Recommended Citation}

Johnson, K. F., Robins, L. B., Williams, C. L., \& Townsend, T. G. (2021). Interrogating systems that cause disparities: testing the social-ecological model in low- versus high-density African American communities. Journal of Social, Behavioral, and Health Sciences, 15(1), 329-344. https://doi.org/10.5590/JSBHS.2021.15.1.22 
Johnson et al., 2021

\section{Introduction}

The African American (AA) community, despite the history of discrimination, historical trauma, and systematic oppression, is making strides in the United States; however, disparities can still be found in mental and physical health outcomes. These disparities are a result of systemic challenges, such as access to care. Access and utilization of care are complicated by (a) sociocultural factors, such as stigma or mistrust of health-care providers; (b) policies that cause high rates of uninsured or underinsured persons (Kawaii-Bogue et al., 2017); and (c) external factors, such as location, transportation, waitlist, and health professional shortages (Campbell et al., 2006; Correll et al., 2011; Planey et al., 2019).

The challenges noted above contribute to the disproportionate economic, physical, and mental health challenges some AAs may incur. To further examine the various levels that influence health-related outcomes, the socialecological model (SEM; Bronfenbrenner, 1977, 1979) was used in the current study. The SEM model framework contextualizes the experiences that can lead to health disparities. To that end, the current research sought to examine (a) the relationship and predictability between high- and low-density AA communities; (b) social and economic factors; (c) physical environment; and (d) poor mental and physical health days. The four-level SEM assists in understanding disparities in health-related outcomes in high- and low-density AA communities.

The first level of the SEM model is the Individual, which is defined as biological and personal histories (i.e., age, education, sex, race, income); the researchers specifically examined race in terms of high- versus lowdensity AA communities. Community-level indicators, such as income, are a predictor of health of members in the community (McLaughlin et al., 2012), and racial diversity of the community is a known predictor of mental and medical health outcomes (Williams et al., 2010). Many community-level studies focus on examining one community in isolation (Davies \& Kelly, 2014); however, the current research study explores the nuances between communities that have high versus low levels of AA populations. This understanding helped to better contextualize the findings and inform practice-based solutions for specific communities. The community based mental health promotion movement was partially driven by the notion of not being able to treat someone in isolation from factors that might be impacting them in their community (Barry, 2019), which includes environmental and personal factors, such as relationships.

The second level of the SEM model is Relationship, defined as social support and close relationships; the researchers specifically examined this indicator in terms of social support, familial support, and the composite variable included economic indicators (i.e., income). The research has demonstrated for various groups of people the relationship between social support and positive physical and mental health outcomes (Blazer, 2005; Holt-Lunstad et al., 2010; Lin et al., 2013; Rothon et al., 2012). Social support is an important variable as it relates to overall well-being and serves as a buffer between predictors and adverse outcomes (Thoits, 2011; Uchino, 2006; Umberson \& Montez, 2010). In addition, social support is highly related to culture (Kim et al., 2008) and ethnicity/race (Klineberg et al., 2006). Lastly, research exists that relates racial/ethnic disparities, social support, and mental health, underscoring the influence of both race and social support on health outcomes, such as depression (Almeida et al., 2011; Shim et al., 2012). Our study focuses on racialethnic differences conceptualized as high- versus low-density AA communities and a composite variable that includes social support.

The third level of the SEM model is Community, defined as settings in which a person frequents, their physical environment, and characteristics of these settings; the researchers specifically examined the physical environment, including air and water quality, housing, and transit. Generally speaking, research has found that the community can be a barrier to seeking health services (Vyavaharkar et al., 2008). It has previously been established that the overall environmental quality, namely air and water quality, in AA communities has been rated significantly lower than other communities (Brooks et al., 2017; VanDerslice, 2011). This research 
study focuses on how the physical environment will be related to other variables of interest, specifically high -density AA communities, poor physical health, and poor mental health.

The fourth level is Societal, defined as broad societal factors (such as health, economics, education, and social policies) that create the climate for health-care disparities; results from levels one through three show the impact of disparities at the societal level. The levels of the social-ecological model are said to be related and assist in explaining health and health disparities.

\section{African American Community and Health Outcomes}

In the current study, "health" refers to both physical and mental health. Although adult AAs are 20\% more likely to report serious psychological distress than White adults, AAs tend to be clinically diagnosed with fewer mental health issues overall (CDC, 2019; OMH, 2016). For AAs who are diagnosed, they are less likely to follow up with treatment and more likely to experience a severe consequence due to their mental illness (Cai \& Robst, 2016). In the literature, it is noted that AAs have significantly less access to mental health services, are more likely to underutilize mental health services, and are less likely to receive necessary mental health services (Chen et al., 2016). In addition, racial and ethnic minorities are more likely to be misdiagnosed and are more likely to receive minimal and poor-quality care when they receive treatment (Carrington, 2006; Chen et al., 2016). Additionally, AAs tend to delay treatment until symptoms are debilitating, which can lead to a more severe diagnosis (Cai \& Robst, 2016; Carpenter-Song et al., 2010; Interian et al., 2013; Satcher, 2000).

When looking at the overall picture of mental health in various racial groups, most minority groups generally experience fewer mental health disorders as compared to Whites. However, minorities often experience a disproportionately higher burden of disability and consequences that result from mental disorders (American Psychiatric Association, n.d.). Further, although the rate of depression is lower in Hispanics (19.6\%) and Blacks (24.6\%) as compared to Whites (34.7\%), depression in these minority groups is likely to be more persistent (Colby \& Ortman, 2015). The disparities in mental health care, access, utilization, and experience of symptoms can be contextually understood through the framework of the SEM; Bronfenbrenner, 1977), which provides four levels that assist in understanding the causes and provide direction on when and where efforts should be made toward intervention. The SEM can also be used to contextualize physical health outcomes.

The status of physical health amongst the AA population highlights grave disparities. The death rate for AAs was generally higher than Whites for heart diseases, stroke, cancer, asthma, influenza and pneumonia, diabetes, HIV/AIDS, and homicide (U.S. Health and Human Services Office of Minority Health, 2019). AA men have the lowest life expectancy and highest mortality rate in the United States (Fried et al., 2003; Xanthos et al., 2010). And the comorbidity of physical chronic diseases with mental health disorders is high, meaning physical and mental health should not be treated separately because they are interrelated (Daré et al., 2019; Gili et al., 2010). One study found that the prevalence rate of individuals having mental health disorders was significantly higher in patients with chronic health diseases (56.8\%) when compared to those who were healthy with no known chronic health diseases (48.9\%; Gili et al., 2010). While this has been brought to the attention of policymakers, and increased prevention efforts have been implemented, there are still mental and physical health disparities between AAs in the United States and non-African Americans. With research highlighting the link between both mental and physical health disparities (Daré et al., 2019; Shim et al., 2013), a framework that allows us to conceptualize both together is important for understanding contributors to mental and physical health and the entry points for interventions that can impact both.

Related to individual physical and mental health is community health. Community health refers to the "health status of a defined group of people and the actions and conditions, both private and public, to promote, protect, and preserve their health" (McKenzie et al., 2005, p. 5). There can be protective factors related to community mental and physical health, such as income, sustainable jobs, perceived social support, and close 
community ties (Ward \& Mengesha, 2013). In addition to these factors, the AA community exhibits certain protective factors, such as religiosity, collective efficacy, social cohesion, and social control (Sharma et al., 2019). There can also be adverse mental and physical health outcomes related to living in a certain community. The literature shows that extreme poverty neighborhoods (i.e., neighborhoods where greater than $40 \%$ of households are below the federal poverty level) experience higher levels of health (e.g., obesity, diabetes, shorter life expectancy) and mental health outcomes (e.g., depression, anxiety, psychological distress) (Acevedo-Garcia et al., 2014; Gupta et al., 2007; Manseau, 2014). Some prior research showed that heavily populated AA communities sometimes face higher disparities in health outcomes because of various social health barriers (Saegert et al., 2011), structural barriers (Johnson \& Bonner, 2013), and personal factors (Signorello et al., 2010).

But research has not compared highly populated AA communities to lowly populated AA communities, and the current study is interested in understanding the health of a community based on its population of AAs. The interest is in understanding if communities with more AA residents have higher disparities. To understand health disparities locally, we included all Virginia counties in the analysis.

\section{Statement of the Problem}

The SEM framework and the four levels of influence assist researchers in understanding why some AA communities may be unduly impacted by health disparities based on social-economic factors, physical environment, and individual factors. As an example, physical environment is adversely correlated with both mental and physical health (Reddy et al., 2019), and the current study further examines this relationship within high- and low-density AA community populations. Likewise, social-economic disparities are adversely related to physical and mental health in the literature, with most studies using poverty level or income (Braveman et al., 2010), which can be problematic as a single indicator (Lantz \& Pritchard, 2010). In seeking to further understand the relationships amongst (a) mental and physical health, (b) social-economic factors, and (c) physical environment, the following research questions guided the study:

Research Question 1: Is there a relationship between poor mental and physical health days; social and economic factors; physical environment; and African American community population density (AACPD)?

Hypothesis 1: There is a relationship between poor mental and physical health days; social and economic factors; physical environment; and AACPD.

Research Question 2: Are there differences between low and high AACPD and poor mental and physical health days; social and economic factors; and physical environment?

Hypothesis 2: High-density African American communities will have more disparities in social and economic factors; physical environment; and poor physical and mental health days.

Research Question 3: Do social and economic factors; physical environment; and AACPD predict poor mental health and physical health days?

Hypothesis 3: Social and economic factors; physical environment; and AACPD are significant predictors of poor mental health days.

Hypothesis 4: Social and economic factors; physical environment; and AACPD are significant predictors of poor physical health days.

The goal of the research study is to understand the relationship between these indicators of health and risk to better inform health-care strategies, interventions, and prevention efforts. 


\section{Method}

This cross-sectional study examined health-related outcomes in high- and low-density AA communities in Virginia. A cross-sectional study is a descriptive study that involves analyzing data from a specific population at a specific point in time; it is specifically suitable when the researcher wants to estimate the prevalence of certain characteristics, behaviors, diseases, intentions, and attitudes in a certain population (Polit \& Beck, 2014; Sedgwick, 2014). This cross-sectional study was utilized in the present study as it can highlight the health-related needs of a population by providing useful information to support planning and innovation in health-care delivery (Polit \& Beck, 2014).

\section{Study Population}

We used the 2017 population data collected by the U.S. Census Bureau in Virginia, which included raw numbers and percentages for the number of AAs in every county in Virginia. There are 133 counties and independent cities in Virginia, and they vary greatly demographically and geographically. For example, some are rural in the heart of the Appalachian Mountains, while others are urban and closer to the Atlantic Ocean (Virginia Tourism Corporation, 2020). We used the percentage of AAs in every county to determine low- and high-density AA counties in Virginia.

When examining the percentage of AAs in counties in Virginia, the "low" and "high" were determined by splitting the data set in half using the median (38.5\%). The lowest percentage of AAs was in Craig County, $0.3 \%$. The highest percentage of AAs was in Petersburg, 76.7\%. Results found that 115 counties were determined to have low-density AA populations, while 18 counties were determined to have high-density AA populations. In terms of basic demographics that are relevant to the current study, we investigated the percent Female and Rural and Non-Rural based on low- and high-density AA counties. Females accounted for an average of $50.66 \%$ of low-density AA counties and an average of 50.35\% of high-density AA counties. Rural communities accounted for an average of $55.75 \%$ of low-density AA counties and an average of $30.71 \%$ of high-density AA counties. Non-Rural communities, which included suburban and urban areas, accounted for an average of $44.25 \%$ of low-density AA counties and an average of $69.29 \%$ of high-density AA counties.

\section{Measures}

Archival data from the 2018 County Health Rankings National (CHRN) data set for Virginia were utilized for the remaining variables. These variables include social and economic factors; physical environment; poor mental health days; and poor physical health days.

\section{Social and Economic Factors.}

This data is ranked from 1-133, with 1 being the best social and economic factors (County: Falls Church) to 133 being the worst social and economic factors (County: Petersburg). The ranked variable is based on individuals in the counties' self-report of education, employment, income, family support, social support, and community safety. The CHRN collected this from various sources:

- $\quad$ Education (EDFacts; American Community Survey)

- Employment (Bureau of Labor Statistics)

- Income (Small Area Income and Poverty Estimates; American Community Survey)

- Family and social support (American Community Survey; County Business Patterns)

- Community safety (Uniform Crime Reporting-FBI; CDC WONDER mortality data)

CHRN used a weighted calculation to determine rankings. 


\section{Physical Environment.}

This variable is ranked from 1-133, with 1 being the best rating (County: Hyland), yielding the best physical environment and 133 being the worst rating (County: Scott), yielding the worst environment. This ranked variable is based on the county report of air quality, water quality, housing, and transit. Several sources were used for this variable:

- $\quad$ Air and water quality (Environmental Public Health Tracking Network; Safe Drinking Water Information System)

- Housing and transit (Comprehensive Housing Affordability Strategy [CHAS] data; American Community Survey)

CHRN used a weighted calculation to determine rankings.

\section{Poor Mental Health Days.}

Poor mental health days were reported according to the average number of mentally unhealthy days reported in the past 30 days, using a question from the Behavioral Risk Factor Surveillance System survey. This calculation is age adjusted. Higher numbers are interpreted to mean more "poor mental health days;" therefore, higher numbers indicate an individual experienced poor mental health days at a greater frequency. Overall, there were 133 valid cases analyzed for the poor mental health days variable; $M=3.68(S D=.32)$, the minimum $=2.81$ and maximum 4.65 .

\section{Poor Physical Health Days.}

Poor physical health days were calculated according to the average number of physically unhealthy days reported in the past 30 days, using a question from the Behavioral Risk Factor Surveillance System. This calculation is age adjusted. Higher numbers are interpreted to mean more "poor physical health days;" therefore, higher numbers indicate an individual experienced poor physical health days at a greater frequency. Overall, there were 133 valid cases analyzed for the poor physical health days variable; $M=3.62(S D=.46)$, the minimum $=2.63$ and maximum 5.02.

\section{African American Community Population Density (AACPD).}

AACPD is a categorical variable that represents low- and high-density AA populations in Virginia counties $(N$ $=133)$. The variable is coded as follows: $\mathrm{O}=$ low-density AA population $(n=115)$ and $1=$ high-density AA population $(n=18)$; the median $(38.5 \%)$ was used to separate the data into low and high density. Data for the variable derived from the 2017 Virginia U.S. Census Bureau info.

\section{Data Analysis}

This study utilized Spearman's rank-order correlation to answer Research Question 1. Spearman's correlation allows you to measure the strength and direction of the association between two variables (Tabachnick \& Fidell, 2013). To answer Research Question 2, an independent $t$-test was conducted. A $t$-test is used to compare the means of two groups. To answer Research Question 3, two multiple regressions analyses were conducted. As a predictive analysis, a $t$-test is used to explain the relationship that exists between two or more independent variables and one continuous, dependent variable (Tabachnick \& Fidell, 2013). All assumptions were satisfied prior to performing the analysis, as (a) linearity was met, (b) independence was established, (c) normal distribution was confirmed, and (d) homogeneity of variance was confirmed. In terms of sample size, the Tabachnik and Fidell (2007, p. 123) formula for calculating sample size was used: $N>50+8 \mathrm{~m}$ (i.e., $\mathrm{m}$ represents the number of independent variables and $\mathrm{N}$ represents the needed sample size); the current study has three independent variables, which equate to a needed sample size of 74 , the current study, $N=133$. 


\section{Results}

\section{Research Question 1: Relationship Amongst Variables of Interest}

Table 1 displays the correlations between social-economic factors, physical environment, poor mental health, poor physical health, and AA community population density (AACPD). Results of the Spearman correlation indicated that there was a significant positive association between AACPD and socio-economic factors (rs(133) $=.45, p<.001)$, poor physical health days $(\mathrm{rs}(133)=.32, p<.001)$, and poor mental health days $(\mathrm{rs}(133)=$ $.23, p<.01$ ); physical environment was not significant. The effect size is represented by the Rho value; to that end the relationship between AA population and variables of interest are as follows: social-economic factors have a small to medium effect size; poor physical health days have a small effect size; and poor mental health days have a small effect size, based on Cohen's $d$ effect size interpretations.

Table 1. Correlation Table With Variables of Interest

\begin{tabular}{llllll}
\hline & $\begin{array}{l}\text { AA } \\
\text { population }\end{array}$ & $\begin{array}{l}\text { Social } \\
\text { economic }\end{array}$ & $\begin{array}{l}\text { Physical } \\
\text { environment }\end{array}$ & $\begin{array}{l}\text { Poor physical } \\
\text { health days }\end{array}$ & $\begin{array}{l}\text { Poor mental } \\
\text { health days }\end{array}$ \\
\hline AA population & - & & & \\
Social economic & $.447^{* *}$ & - & & \\
Physical environment & .159 & .138 & - & - \\
$\begin{array}{l}\text { Poor physical health } \\
\text { days }\end{array}$ & $.324^{* *}$ & $.882^{* *}$ & .121 & & - \\
Poor mental health days & $.225^{* *}$ & $.805^{* *}$ & .360 & $.929^{* *}$ & \\
\hline
\end{tabular}

Note. $P$ value: ${ }^{* *}=p<.01$

\section{Research Question 2: Mean Differences of Low- and High-Density AA Communities}

The results from four independent $t$-tests are noted in Table 2. Group one includes $n=115$ counties and indicates low-density AA population in the counties; $n=18$ counties represent the high-density AA population in the counties. $t$-test comparing the means between high and low density and social-economic factors, physical environment, poor physical health days, and poor mental health days, were all significant at the .05 level or less. Cohen's $d$ was used to calculate the effect size for the comparison between two means. In terms of relative size, a small effect size is .2; medium is .5; and large is .8 and above (Cohen, 1992). The formula used was Effect size $=[$ Mean of experimental group $]-[$ Mean of control group $] /$ Standard deviation . 
Table 2. T-Test Results and Means for Groups

\begin{tabular}{lllll}
\hline \multicolumn{5}{c}{ African American Population Community Density } \\
\hline & Low $(n=115)$ & High $(n=18)$ & $t$-Test & Effect size \\
Socio-economic & $60.21(36.22)$ & $110.39(20.70)$ & $t(131)=8.45, p=.000$ & 1.70 \\
Physical environment & $64.58(39.33)$ & $82.44(29.36)$ & $t(131)=2.28, p=.031$ & 0.51 \\
Poor Physical Health & $3.56(.45)$ & $4.00(.38)$ & $t(131)=4.36, p=.000$ & 1.05 \\
Poor Mental Health & $3.66(.33)$ & $3.86(.23)$ & $t(131)=3.23, p=.003$ & 0.70 \\
\hline
\end{tabular}

\section{Research Question 3: Predicting Poor Physical and Mental Health Days}

\section{Predicting Poor Physical Health Days}

Standard multiple regression was used to assess the ability of socio-economic factors, physical environment, and AA community population density to predict poor physical health days. Preliminary analysis was conducted to ensure no violations of the assumptions of normality, linearity, homoscedasticity, and multicollinearity. In the preliminary analysis, multicollinearity was a concern because poor physical health days and social and economic factors were highly correlated $r=.852$. The adjusted $R^{2}$ was used to control for the potential overestimate of the population resulting from high collinearity (Tabachnick \& Fidell, 2013). Results of the multiple linear regression indicated that there was a collective significant effect between socialeconomic factors, physical environment, and AA population, $\left(F(3,129)=117.27, p<.001, R^{2}=.73\right)$. The individual predictors were examined further and indicated that social-economic factors $(t=17.30, p<.001)$ were the only significant predictor in the model.

Table 3. Multiple Regression Predicting Poor Physical Health

\begin{tabular}{|c|c|c|c|c|c|c|c|}
\hline \multirow[b]{2}{*}{ Predictor } & \multirow[t]{2}{*}{ Zero-order } & \multirow[t]{2}{*}{$\mathrm{B}$} & \multirow[t]{2}{*}{ SE B } & \multirow[t]{2}{*}{$B$} & \multicolumn{2}{|c|}{$95 \% \mathrm{CI}$ for $\mathrm{p}$} & \multirow[t]{2}{*}{$t$} \\
\hline & & & & & Lower & Upper & \\
\hline Social-economic factors & .852 & .011 & .001 & .855 & .010 & .012 & $17 \cdot 30^{* *}$ \\
\hline Physical environment & .133 & .000 & .001 & .023 & -.001 & .001 & .497 \\
\hline AA population & .320 & -.109 & .070 & -.080 & -.247 & .030 & -1.55 \\
\hline Adjusted $\mathrm{R}^{2}=.73$ & & & & & & & \\
\hline
\end{tabular}

\section{Predicting Poor Mental Health Days}

Standard multiple regression was used to assess the ability of socio-economic factors, physical environment, and AA community population density to predict poor physical health days. Preliminary analysis was conducted to ensure no violations of the assumptions of normality, linearity, homoscedasticity, and multicollinearity. Results of the multiple linear regression indicated a collective significant effect between 
social-economic factors, physical environment, and AA population, $(\mathrm{F}(3,129)=75.16, p<.001, \mathrm{R} 2=.64)$. The individual predictors were examined further and indicated that social-economic factors $(t=14.41, p<.001)$ and AA community population density $(t=-2.78, p<.001)$ were significant predictors in the model. Physical environment did not significantly contribute to the model.

Table 4. Multiple Regression Predicting Poor Mental Health Days

\begin{tabular}{|c|c|c|c|c|c|c|c|}
\hline \multirow[b]{2}{*}{ Predictor } & \multirow[t]{2}{*}{ Zero-order } & \multirow[t]{2}{*}{ B } & \multirow[t]{2}{*}{ SE B } & \multirow[t]{2}{*}{$\beta$} & \multicolumn{2}{|c|}{$95 \% \mathrm{CI}$ for $\mathrm{p}$} & \multirow[t]{2}{*}{$t$} \\
\hline & & & & & Lower & Upper & \\
\hline Social-economic factors & .784 & .007 & .001 & .858 & .006 & .008 & $14.41^{* *}$ \\
\hline Physical environment & .092 & $>-.000$ & .000 & .000 & -.001 & .001 & -.007 \\
\hline AA population & .217 & -.159 & .057 & -.166 & -.272 & -.046 & $-2.78^{* *}$ \\
\hline$R^{2}=.64$ & & & & & & & \\
\hline
\end{tabular}

Note. $P$ value: ${ }^{* *}=p<.01$

\section{Discussion}

Three research questions sought to understand the relationship and the predictability of AA community, including: (a) population density, (b) social-economic factors, (c) physical environment, and (d) poor physical and mental health days. The goal of this study was to understand the relationship between indicators of health risk and AA community density to better inform health-care strategies, interventions, and prevention efforts. Multiple regression, an independent samples $t$-test, and Spearman's rank correlation were utilized to explore the three proposed research questions. The four-level SEM; Bronfenbrenner, 1977, 1979) guided the exploration of results to understand health disparities between communities with high- and low-density AA populations.

To test the hypothesis that there is a relationship between the variables represented in the four-level social ecological model and AA community population density (AACPD), a Spearman correlation was utilized. The results indicate that there is a positive association between AACPD and socio-economic factors and poor physical and mental health days, with small to medium effect sizes. The positive association between AACPD and socio-economic factors is consistent with previous literature that investigates race, socio-economic status, and health showing an adverse relationship (William et al., 2010, 2016). The positive associations between AACPD and poor physical and mental health days are well represented in the literature, as AAs experience revolving, significant mental and physical health issues that are often co-occurring (Shim et al., 2013). More specifically, with regard to poor mental health days, AAs reported experiencing higher rates of feelings of sadness (4.3\%) and hopelessness (2.1\%), as compared to Whites (2.6\% and 1.9\%, respectively) (CDC, 2019). What is unique in the findings is the absence of an association between AACPD and physical environment. Research consistently notes the documentation of significantly lower environmental quality (Brooks et al., 2017) and poor air quality (Mikati et al., 2018) in high-density AA communities.

To test the hypothesis that high-density AA communities will have more disparities in social-economic factors, physical environment, and poor physical and poor mental health days (Hypothesis 2), an independent $t$-test was used to compare the means of high and low AA community density groups. All the mean differences 
were significant, with medium to large effect sizes. In the high-density AA communities' group, the mean was higher for social-economic factors, with a large effect size, interpreted as a significant finding indicating more social-economic challenges in that group. These findings are in line with prior research that shows economic disparities between AAs and White families (Reardon \& Bischoff, 2011; Shapiro et al., 2013). Physical environment-a composite variable that assessed air quality, water purity, and pollution -was higher (i.e., negative) in the high-density AA group with a medium effect size. The SEM assists in interpreting the relative importance of this finding; it highlights the relationship between health disparities and physical environment. This finding is also in line with prior research that shows AAs tend to rate environmental quality (i.e., air and water quality) significantly lower than their non-AA counterparts (Brooks et al., 2017; VanDerslice, 2011). In terms of highly populated AA communities, some research has even noted that these communities are in areas that contain poor air quality due to health-threatening air pollution (Mikati et al., 2018), which is an environmental health equity issue because the adverse health outcomes related to poor physical environment are well known (Mikati et al., 2018). In terms of the mean differences between low- and high-density AA community density and poor physical health mental health days, the means are very close but there is a significant difference with a large and medium effect size, respectively. The mean scores are slightly higher in the high AA group; however, based on the research literature, the expectations were larger mean differences (Dwyer-Lindgren et al., 2017). For both groups, mean scores were close to the overall mean, which means neither group reported a higher-than-normal amount of poor physical and mental health days.

To test the hypothesis that poor physical health (Hypothesis 3) and mental health days (Hypothesis 4) can be predicted by indicators in the four-level social ecological model, two multiple regression analyses were conducted. Both models included three predictors that represented level 1 of the SEM (i.e., AA population community density), level 2 of the SEM (i.e., social and economic factors), and level 3 (i.e., physical environment). Physical environment was not significant in any of the models. The largest contributor to explaining the variance found in poor physical health days (beta $=.855$ ) and poor mental health days (beta $=$ .858) was social and economic factors explaining $86 \%$ of the variance. This means that the most important contributor to poor physical and mental health days above and beyond AA community density was social and economic factors. A recent study investigating geographic disparities found that $60 \%$ of the variability in life expectancy can be attributed to social-economic factors and race/ethnicity factors (Dwyer-Lindgren et al., 2017). While the study is not the same, it does show the influential nature of social and economic factors on health (Dwyer-Lindgren et al., 2017).

In our current study, we examined level one of the SEM through the dummy variable high and low AA community density (i.e., variable in the model labeled "AA population") because we were particularly interested in disparities amongst the communities. Some prior research showed that heavily populated AA communities sometimes face higher disparities in health outcomes because of various social health barriers (Saegert et al., 2011), structural barriers (Johnson \& Bonner, 2013), and personal factors (Signorello et al., 2010), but research has not compared highly populated AA communities to lowly populated AA communities. Inferences can be made based on the current research on AA and White American disparities (Snowden, 2012), but current researchers wanted to test the hypothesis that there were differences based on AA community density. However, in our study, AA population was not a significant contributor to the model predicting poor physical health, but it was a significant contributor to the model predicting poor mental health (beta $=-.166$ ). The negative beta indicates that Group 1 low-density AA community (coded as 0 ) is a stronger contributor to the significant contribution to predicting poor mental health days. This finding is interesting and is related to the literature that finds non-White people are diagnosed with fewer mental health challenges than their White counterparts (Brody et al., 2018). It may also be reminiscent of research that highlights that non-White persons are severely underdiagnosed for mental health challenges, and the research that shows that non-White people may attribute mental health challenges to other external causes, such as stress, finances, etc. (Brody et al., 2018; Pratt \& Brody, 2014). 
Improving the amount of physically and mentally healthy days a person experiences is a benchmark that insurance companies, national health organizations, and stakeholders in population health often use to gauge the success of health promotion prevention and intervention efforts (Cordier et al., 2018). Our research highlights that an important predictor of healthy days is social-economic factors. According to the SEM, individual and relationship indicators are important in understanding and explaining health disparities (Bronfenbrenner, 1977). Our research is in line with the SEM theory, in that components at the individual level (i.e., economic factors) and components at the relationship level (i.e., social support, familial support, etc.), which was a composite variable in the current study, both contribute to physical and mental health days (Nategh et al., 2017).

There are a few limitations within this study that should be mentioned. This study used pre-existing data from a reputable source, although the researchers did not oversee the data collection process. Although the data was readily available, this limited the type of collected data that could be useful within this study. Most of this data was self-reported, allowing room for reporter bias. This research only explored counties within Virginia, limiting the generalizability of this study. The limitations of this cross-sectional design are (a) inability to measure incidence, (b) causation can't be implied, (c) identified associations are difficult to interpret, (d) inaccurate self-reporting to surveys, and (e) susceptible to biases. (Connelly, 2016; Sedgwick, 2014). Although limitations are known, this design is beneficial because multiple outcomes can be studied, useful for descriptive analyses, and useful with many populations. (Polit \& Beck, 2014).

\section{Implications for Behavioral Health Providers}

Our research highlights the disproportionate risk high-density AA communities may face at all levels of the social ecological framework. The SEM was used to understand health disparities, and what influences disparities at various levels of influence. Our cumulative findings suggest that models of care that are holistic, that minimize social and economic barriers to care, would be best in supporting the overall health of people living in high-density AA communities.

Integrated behavioral health (IBH) care is a model of care that focuses on minimizing barriers to health-care access through synchronized health-care services, integrating both behavioral and medical services (Peek, 2011). This concept was established in the 1970 s to ensure everyone had access to mental health care (Coleman \& Patrick, 1976); since, proponents of this model have consistently noted its success in serving vulnerable and underserved populations by increasing access to mental and physical health services (MillerMatero et al., 2016; Ohrnberger et al., 2017). Utilizing the IBH model allows clients to access multiple services in one location, providing a comprehensive approach to health care (Perez-Brumer et al., 2016). This model is also considered a best practice for working with clients who require both medical and behavioral health services (Miller-Matero et al., 2016). Our study found that in counties where the population of AAs is the highest, the respondents indicate higher needs related to social-economic factors and physical and mental

health. IBH is a reasonable and evidence-based practice that can meet the needs of persons in these counties (Miller-Matero et al., 2016). 


\section{References}

2018 County Health Rankings and Roadmaps. (2021). Rankings data and documentation: National data and documentation: 2021 county health rankings. https://www.countyhealthrankings.org/explorehealth-rankings/rankings-data-documentation

Acevedo-Garcia, D., McArdle, N., Hardy, E. F., Crisan, U. I., Romano, B., Norris, D., Baek, M., \& Reece, J. (2014). The child opportunity index: Improving collaboration between community development and public health. Health Affairs, 33(11), 1948-1957. https://doi.org/10.1377/hlthaff.2014.0679

Almeida, J., Subramanian, S. V., Kawachi, I., \& Molnar, B. E. (2011). Is blood thicker than water? Social support, depression and the modifying role of ethnicity/nativity status. Journal of Epidemiology and Community Health, 65(1), 51-56. https://doi.org/10.1136/jech.2009.092213

American Psychiatric Association. (n.d.). Mental health disparities: Diverse populations. https://www.psychiatry.org/psychiatrists/cultural-competency/education/mental-health-facts

Barry, M. M., Clarke, A. M., Petersen, I., \& Jenkins, R. (2019). Community mental health promotion principles and strategies. In Implementing Mental Health Promotion (2nd ed., pp. 163-194). Springer.

Blazer, D. G. (2005). Depression and social support in late life: A clear but not obvious relationship. Aging and Mental Health, 9(6): 497-499. https://doi.org/10.1080/13607860500294266

Braveman, P. A., Cubbin, C., Egerter, S., Williams, D. R., \& Pamuk, E. (2010). Socioeconomic disparities in health in the United States: What the patterns tell us. American Journal of Public Health, 10o(s1), S186-S196. https://doi.org/10.2105/AJPH.2009.166082

Brody, D. J., Pratt, L. A., \& Hughes, J. P. (2018). Prevalence of depression among adults aged 20 and over: United States, 2013-2016 (NCHS Data Brief No. 303). National Center for Health Statistics.

Bronfenbrenner, U. (1977). Toward an experimental ecology of human development. American Psychologist, 32(7), 513-531. https://doi.org/10.1037/0003-066X.32.7.513

Bronfenbrenner, U. (1979). The ecology of human development: Experiments by nature and design. Harvard University Press.

Brooks, C. J., Gortmaker, S. L., Long, M. W., Cradock, A. L., \& Kenney, E. L. (2017). Racial/ethnic and socioeconomic disparities in hydration status among U.S. adults and the role of tap water and other beverage intake. American Journal of Public Health, 107(9), 1387-1394. https://doi.org/10.2105/AJPH.2017.303923

Cai, A., \& Robst, J. (2016). The relationship between race/ethnicity and the perceived experience of mental health care. American Journal of Orthopsychiatry, 86(5), 508-518. https://doi.org/10.1037/ortoooo119

Campbell, C. D., Kearns, L. A., \& Patchin, S. (2006). Psychological needs and resources as perceived by rural and urban psychologists. Professional Psychology: Research and Practice, 37(1), 45-50. https://doi.org/10.1037/0735-7028.37.1.45

Carpenter-Song, E., Chu, E., Drake, R. E., Ritsema, M., Smith, B., \& Alverson, H. (2010). Ethno-cultural variations in the experience and meaning of mental illness and treatment: Implications for access and utilization. Transcultural Psychiatry, 47(2), 224-251. https://doi.org/10.1177\%2F1363461510368906

Carrington, C. H. (2006). Clinical depression in African American women: Diagnoses, treatment, and research. Journal of Clinical Psychology, 62(7), 779-791. https://doi.org/10.1002/jclp.20289 
Centers for Disease Control and Prevention. (2019). Summary Health Statistics: National Health Interview Survey: 2018. Table A-7. https://ftp.cdc.gov/pub/Health_Statistics/NCHS/NHIS/SHS/2018_SHS_Table_A-7.pdf

Chen, J., Vargas-Bustamante, A., Mortensen, K., \& Ortega, A. N. (2016). Racial and ethnic disparities in health care access and utilization under the Affordable Care Act. Medical Care, 54(2), 140-146. https://doi.org/10.1097\%2FMLR.0000000000000467

Cohen, J. (1992). A power primer. Psychological Bulletin, 112(1), 155-159. https://doi.org/10.1037//o0332909.112.1.155

Colby, S. L., \& Ortman, J. M. (2015). Projections of the size and composition of the US population: 2014 to 2060: Population estimates and projections (Report No. P25-1143). https://www.census.gov/content/dam/Census/library/publications/2015/demo/p25-1143.pdf

Coleman, J. V., \& Patrick, D. L. (1976). Integrating mental health services into primary medical care. Medical Care, 14(8), 654-661. https://doi.org/10.1097/00005650-197608000-00002

Connelly, L. M. (2016, September-October). Cross-sectional survey research. MedSurg Nursing, 25(5), 369370.

Cordier, T., Song, Y., Cambon, J., Haugh, G. S., Steffen, M., Hardy, P., Staehly, M., Hagan, A., Gopal, V., Tye, P. D., \& Renda, A. (2018). A bold goal: More healthy days through improved community health. Population Health Management, 21(3), 202-208. https://doi.org/10.1089/pop.2017.0142

Correll, J. A., Cantrell, P., \& Dalton, W. T., III. (2011). Integration of behavioral health services in a primary care clinic serving rural Appalachia: Reflections on a clinical experience. Families, Systems, and Health, 29(4), 291-302. https://doi.org/10.1037/a0026303

Daré, L. O., Bruand, P.-E., Gérard, D., Marin, B., Lameyre, V., Boumédiène, F., \& Preux, P.-M. (2019). Comorbidities of mental disorders and chronic physical diseases in developing and emerging countries: A meta-analysis. BMC Public Health, 19(1), Article 304. https://doi.org/10.1186/s12889-019-6623-6

Davies, J. K., \& Kelly, M. (Eds.). (2014). Healthy cities: Research and practice. Routledge.

Dwyer-Lindgren, L., Bertozzi-Villa, A., Stubbs, R. W., Morozoff, C., Mackenbach, J. P., van Lenthe, F. J., Mokdad, A. H., \& Murray, C. J. L. (2017). Inequalities in life expectancy among U.S. counties, 1980 to 2014: Temporal trends and key drivers. JAMA Internal Medicine, 177(7), 1003-1011. https://doi.org/10.1001/jamainternmed.2017.0918

Fried, V. M., Prager, K., MacKay, A. P., Xia, H. (2003). Chartbook on trends in the health of Americans. Health, United States, 2003. National Center for Health Statistics. https://www.cdc.gov/nchs/data/hus/huso3.pdf

Gili, M., Comas, A., García-García, M., Monzón, S., Antoni, S.-B., \& Roca, M. (2010). Comorbidity between common mental disorders and chronic somatic diseases in primary care patients. General Hospital Psychiatry, 32(3), 240-245. https://doi.org/10.1016/j.genhosppsych.2010.01.013

Gupta, R. P.-S., de Wit, M. L., \& McKeown, D. (2007). The impact of poverty on the current and future health status of children. Paediatrics and Child Health, 12(8), 667-672. https://doi.org/10.1093/pch/12.8.667

Holt-Lunstad, J., Smith, T. B., \& Layton, J. B. (2010). Social relationships and mortality risk: A meta-analytic review. PLoS Medicine, 7(7), e1000316. https://doi.org/10.1371/journal.pmed.1000316

Interian, A., Lewis-Fernández, R., \& Dixon, L. B. (2013). Improving treatment engagement of underserved U.S. racial-ethnic groups: A review of recent interventions. Psychiatric Services, 64(3), 212-222. https://doi.org/10.1176/appi.ps.201100136 
Johnson, K., \& Bonner, M. (2013). Utilizing the six generic human service competencies and ecological systems theory as a basis to understanding barriers faced by marginalized clients. Journal of Human Services, 33(1). https://digitalcommons.odu.edu/chs_pubs/31/

Kawaii-Bogue, B., Williams, N. J., \& MacNear, K. (2017). Mental health care access and treatment utilization in African American communities: An integrative care framework. Best Practices in Mental Health, 13(2), 11-29.

https://www.ingentaconnect.com/content/follmer/bpmh/2017/oooooo13/00000002/artooo04

Kim, H. S., Sherman, D. K., \& Taylor, S. E. (2008). Culture and social support. American Psychologist, 63(6), 518-526. https://doi.org/10.1037/0003-066X

Klineberg, E., Clark, C., Bhui, K. S., Haines, M. M., Viner, R. M., Head, J., Woodley-Jones, D., \& Stansfeld, S. A. (2006). Social support, ethnicity and mental health in adolescents. Social Psychiatry and Psychiatric Epidemiology, 41(9), 755-760. https://doi.org/10.1007/s00127-006-0093-8

Lantz, P. M., \& Pritchard, A. (2010). Socioeconomic indicators that matter for population health. Preventing Chronic Disease, 7(4). https://www.cdc.gov/pcd/issues/2010/jul/o9_0246.htm

Lin, N., Dean, A., \& Ensel, W. M. (Eds.). (2013). Social support, life events, and depression. Academic Press.

Manseau, M. W. (2014). Economic inequality and poverty as social determinants of mental health. Psychiatric Annals, 44(1), 32-38. https://doi.org/10.3928/00485713-20140108-06

McKenzie, J. F., Pinger, R. R., \& Kotecki, J. E. (2005). An Introduction to Community Health. Jones \& Bartlett Learning.

McLaughlin, K. A., Costello, E. J., Leblanc, W., Sampson, N. A., \& Kessler, R. C. (2012). Socioeconomic status and adolescent mental disorders. American Journal of Public Health, 102(9), 17421750. https://doi.org/10.2105/AJPH.2011.300477

Mikati, I., Benson, A. F., Luben, T. J., Sacks, J. D., \& Richmond-Bryant, J. (2018). Disparities in distribution of particulate matter emission sources by race and poverty status. American Journal of Public Health, 108(4), 480-485. https://doi.org/10.2105/AJPH.2017.304297

Miller-Matero, L. R., Dykuis, K. E., Albujoq, K., Martens, K., Fuller, B. S., Robinson, V., \& Willens, D. E. (2016). Benefits of integrated behavioral health services: The physician perspective. Families, Systems, and Health, 34(1), 51-55. https://doi.org/10.1037/fshooo0182

Nategh, A., Malek, A., Sadegh, H., \& Davoud, A. (2017). Women physical activity and its effective factors using a social-ecological model. Annals of Medical and Health Sciences Research, 7.

https://www.amhsr.org/articles/women-physical-activity-and-its-effective-factors-usingasocialecological-model-4021.html

Peek, C. J. (2011). A collaborative care lexicon for asking practice and research development questions. In C. Mullican (Ed.), A national agenda for research in collaborative care: Papers from the Collaborative Care Research Network Research Development Conference (AHRQ Publication No. 11-0067, pp. 2544). Agency for Healthcare Research and Quality. https://archive.ahrq.gov/research/findings/finalreports/collaborativecare/collabcare.pdf

Perez-Brumer, A. G., Oldenburg, C. E., Reisner, S. L., Clark, J. L., \& Parker, R. G. (2016). Towards "reflexive epidemiology": Conflation of cisgender male and transgender women sex workers and implications for global understandings of HIV prevalence. Global Public Health, 11(7-8), 849-865. https://doi.org/10.1080/17441692.2016.1181193

Planey, A. M., Smith, S. M., Moore, S., \& Walker, T. D. (2019). Barriers and facilitators to mental health helpseeking among African American youth and their families: A systematic review study. Children and Youth Services Review, 101, 190-200. https://doi.org/10.1016/j.childyouth.2019.04.001 
Polit, D. F., \& Beck, C. T. (2014). Essentials of nursing research: Appraising the evidence for nursing practice. Wolters Kluwer/Lippincott Williams \& Wilkins.

Pratt, L. A., \& Brody, D. J. (2014). Depression in the U.S. household population, 2009-2012. NCHS Data Brief, 172, 1-8. https://www.cdc.gov/nchs/data/databriefs/db172.pdf

Reardon, S. F., \& Bischoff, K. (2011). Income inequality and income segregation. American Journal of Sociology, 116(4), 1092-1153. https://doi.org/10.1086/657114

Reddy, K. R., Cameselle, C., \& Adams, J. A. (2019). Sustainable engineering: Drivers, metrics, tools, and applications. John Wiley \& Sons.

Rothon, C., Goodwin, L., \& Stansfeld, S. (2012). Family social support, community “social capital” and adolescents' mental health and educational outcomes: a longitudinal study in England. Social Psychiatry and Psychiatric Epidemiology, 47(5), 697-709. https://doi.org/10.1007/s00127-0110391-7

Saegert, S., Fields, D., \& Libman, K. (2011). Mortgage foreclosure and health disparities: Serial displacement as asset extraction in African American populations. Journal of Urban Health, 88(3), 390-402. https://doi.org/10.1007/s11524-011-9584-3

Satcher, D. S. (2000). Executive summary: A report of the Surgeon General on mental health. Public Health Reports, 115(1), 89-101. https://doi.org/10.1093/phr/115.1.89

Sedgwick, P. (2014). Cross sectional studies: Advantages and disadvantages. BMJ, 2014(348), g2276. https://doi.org/10.1136/bmj.g2276

Shapiro, T., Meschede, T., \& Osoro, S. (2013, February). The roots of the widening racial wealth gap: Explaining the Black-White economic divide (Research and Policy Brief). IASP. https://heller.brandeis.edu/iere/pdfs/racial-wealth-equity/racial-wealth-gap/roots-widening-racialwealth-gap.pdf

Sharma, S., Mustanski, B., Dick, D., Bolland, J., \& Kertes, D. A. (2019). Protective factors buffer life stress and behavioral health outcomes among high-risk youth. Journal of Abnormal Child Psychology, 47(8), 1289-1301. https://doi.org/10.1007/s10802-019-00515-8

Shim, R. S., Baltrus, P., Bradford, L. D., Holden, K. B., Fresh, E., \& Fuller, L. E. (2013). Characterizing depression and co-morbid medical conditions in African American women in a primary care setting. Journal of the National Medical Association, 105(2), 183-191. https://doi.org/10.1016/So0279684(15)30106-1

Shim, R. S., Ye, J., Baltrus, P., Fry-Johnson, Y., Daniels, E., \& Rust, G. (2012, Winter). Racial/ethnic disparities, social support, and depression: examining a social determinant of mental health. Ethnicity and Disease, 22(1), 15-20. https://www.ncbi.nlm.nih.gov/pmc/articles/PMC4039297/

Signorello, L. B., Hargreaves, M. K., \& Blot, W. J. (2010). The southern community cohort study: investigating health disparities. Journal of Health Care for the Poor and Underserved, 21(1), 26-37. https://doi.org/10.1353/hpu.0.0245

Snowden, L. R. (2012). Health and mental health policies' role in better understanding and closing African American-White American disparities in treatment access and quality of care. American Psychologist, 67(7), 524-531. https://doi.org/10.1037/a0030054

Tabachnick, B. G., \& Fidell, L. S. (2013). Using multivariate statistics (6th ed). Pearson.

Thoits, P. A. (2011). Mechanisms linking social ties and support to physical and mental health. Journal of Health and Social Behavior, 52(2), 145-161. https://doi.org/10.1177/0022146510395592 
Uchino, B. N. (2006). Social support and health: A review of physiological processes potentially underlying links to disease outcomes. Journal of Behavioral Medicine, 29(4), 377-387. https://doi.org/10.1007/s10865-006-9056-5

Umberson, D., \& Montez, J. K. (2010). Social relationships and health: A flashpoint for health policy. Journal of Health and Social Behavior, 51(1_suppl), S54-S66. https://doi.org/10.1177/0022146510383501

U. S. Census Bureau. (2018). American Community Survey 2013-2017 5-year data release (Multimedia). https://www.census.gov/newsroom/press-kits/2018/acs-5year.html

U.S. Department of Health and Human Services Office of Minority Health. (2019). Profile: Black/African Americans [Interactive media]. https://www.minorityhealth.hhs.gov/omh/browse.aspx?lvl=3\&lvlid=61

VanDerslice, J. (2011). Drinking water infrastructure and environmental disparities: Evidence and methodological considerations. American Journal of Public Health, 101(Suppl. 1), S109-S114. https://doi.org/10.2105/AJPH.2011.300189

Virginia Tourism Corporation. (2021). Virginia places to visit. https://www.virginia.org/places-to-visit/

Vyavaharkar, M. V., Moneyham, L., \& Corwin, S. (2008). Health care utilization: The experiences of rural HIV-positive African American women. Journal of Health Care for the Poor and Underserved, 19(1), 294-306. https://doi.org/10.1353/hpu.2008.0013

Ward, E., \& Mengesha, M. (2013). Depression in African American men: A review of what we know and where we need to go from here. American Journal of Orthopsychiatry, 83(2-3), 386-397. https://doi.org/10.1111/ajop.12015

Williams, D. R., Mohammed, S. A., Leavell, J., \& Collins, C. (2010). Race, socioeconomic status and health: Complexities, ongoing challenges and research opportunities. Annals of the New York Academy of Sciences, 1186(1), 69-101. https://doi.org/10.1111/j.1749-6632.2009.05339.x

Xanthos, C., Treadwell, H. M., \& Holden, K. B. (2010). Social determinants of health among African-American men. Journal of Men's Health, 7(1), 11-19. https://doi.org/10.1016/j.jomh.2009.12.002

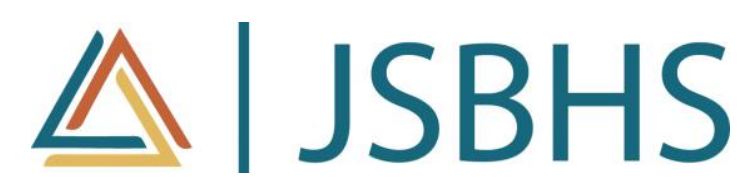

The Journal of Social, Behavioral, and Health Sciences (JSBHS), cosponsored by the College of Health Professionals and the College of Social and Behavioral Sciences at Walden University, is a peer-reviewed, online, interdisciplinary journal focusing on theoretically based research that addresses contemporary national and international issues. $J S B H S$ articles include peer-reviewed research reports, brief reports, comprehensive literature reviews, book reviews, and student research. 